\title{
Neonatal sepsis at Muhimbili National Hospital, Dar es Salaam, Tanzania; aetiology, antimicrobial sensitivity pattern and clinical outcome
}

Tumaini V Mhada', Francis Fredrick ${ }^{2 *}$, Mecky I Matee ${ }^{3}$ and Augustine Massawe ${ }^{2}$

\begin{abstract}
Background: Neonatal sepsis contributes significantly to morbidity and mortality among young infants. The aetiological agents as well as their susceptibility to antimicrobial agents are dynamic. This study determined aetiology, antimicrobial susceptibility and clinical outcome of neonatal sepsis at Muhimbili National Hospital.

Methods: Three hundred and thirty neonates admitted at the Muhimbili National Hospital neonatal ward between October, 2009 and January, 2010 were recruited. Standardized questionnaires were used to obtain demographic and clinical information. Blood and pus samples were cultured on MacConkey, blood and chocolate agars and bacteria were identified based on characteristic morphology, gram stain appearance and standard commercially prepared biochemical tests. Antimicrobial sensitivity testing was performed for ampicillin, cloxacillin, gentamicin, amikacin, cefuroxime and ceftriaxone on Mueller Hinton agar using the Kirby Bauer diffusion method.
\end{abstract}

Results: Culture proven sepsis was noted in 24\% (74/330) of the study participants. Isolated bacterial pathogens were predominantly Staphylococcus aureus, Klebsiella spp and Escherichia coli. Klebsiella spp 32.7\% (17/52) was the predominant blood culture isolate in neonates aged below seven days while Staphylococcus aureus 54.5\% (12/22) was commonest among those aged above seven days. Staphylococcus aureus was the predominant pus swabs isolate for both neonates aged 0-6 days 42.2\% (98/232) and 7-28 days 52.3\% (34/65). Resistance of blood culture isolates was high to ampicillin $81.1 \%$ (60/74) and cloxacillin 78.4\% (58/74), moderate to ceftriaxone $14.9 \%(11 / 74)$ and cefuroxime $18.9 \%(14 / 74)$, and low to amikacin 1.3\% (1/74). Isolates from swabs had high resistance to ampicillin 89.9\% (267/297) and cloxacillin 85.2 (253/297), moderate resistance to ceftriaxone 38.0\% (113/297) and cefuroxime 36.0\% (107/297), and low resistance to amikacin 4.7\% (14/297). Sepsis was higher in neonates with fever and hypothermia $(p=0.02)$, skin pustules $(p<0.001)$, umbilical pus discharge and abdominal wall hyperemia $(p=0.04)$. Presence of skin pustules was an independent predictor of sepsis OR $0.26,95 \% \mathrm{Cl}(0.10-0.66) \mathrm{p}=0.004$. The overall death rate was 13.9\% (46/330), being higher in neonates with sepsis $24.3 \%$ (18/74) than those without $10.9 \%$ (28/256), $p=0.003$.

Conclusions: Staphylococcus aureus was predominant isolate followed by Klebsiella and Escherichia coli. There was high resistance to ampicillin and cloxacillin. Mortality rate due to neonatal sepsis was high in our setting. Routine antimicrobial surveillance should guide the choice of antibiotics for empirical treatment of neonatal sepsis.

\footnotetext{
*Correspondence: fredrick.francis78@gmail.com

${ }^{2}$ Department of Paediatrics and Child Health, School of Medicine, Muhimbili University of Health and Allied Sciences, P O Box 65001, Dar es Salaam, Tanzania

Full list of author information is available at the end of the article
} 


\section{Background}

Neonatal mortality contribute significantly to the infant mortality rates in developing countries, various conditions are responsible for neonatal mortality among which is neonatal sepsis, which account for about $26 \%$ of neonatal mortality [1]. About four million neonatal deaths occur worldwide with reported highest rates in Sub-Sahara Africa [1].

Neonatal sepsis which is defined as sepsis occurring in the first 28 days of life can be divided into early onset and late onset. Differentiation into early and late onset neonatal sepsis is important in prevention and treatment because of aetiological differences. Very few studies in sub-Sahara Africa has looked into neonatal sepsis with the focus on time of onset [2,3]. In this study we defined early onset neonatal bacterial sepsis as sepsis in neonates less than seven days and late onset as sepsis in 7 days or more.

Clinical presentation of neonatal sepsis varies and there are no pathognomonic features [4], however some clinical features have been reported to predict sepsis. Kayange et al. in a study which was conducted in Bugando, Tanzania reported inability to breast feed, lethargy, convulsion, chest wall in-drawing, jaundice and umbilical redness to be strongly associated with neonatal sepsis [5]. Non-specific presentation of neonatal sepsis and poor or delayed laboratory services have resulted in empirical treatment of sepsis in resource limited set up. In a study conducted in Kilimanjaro, Tanzania more than two thirds of neonates admitted to a special care baby unity received antibiotics [6].

In the management of neonatal sepsis, clinicians in many resource limited settings make tentative diagnosis and empirical treatment of neonatal sepsis based on the new neonatal WHO Integrated Management of Childhood Illnesses (n-IMCI) guidelines [7]. However aetiology of neonatal sepsis as well as response to antimicrobial agents may vary significantly from time to time and geographically which may affect the success of empirical management [5,7-10].

Currently at Muhimbili National Hospital, the largest referral hospital in Tanzania, neonatal sepsis is managed according to WHO guideline which recommends ampicillin, cloxacillin and gentamycin as the first line antibiotics [11]. Reports of dynamic nature of the aetiological agents and their response to antimicrobial agents in different geographical areas calls for availability of local data in guiding choices of antibiotics. This study was carried out to determine aetiology, antimicrobial resistance of isolated bacteria and outcome of neonatal sepsis.

\section{Methods}

This was a hospital based cross-sectional study carried out at Muhimbili National Hospital neonatal unit. Both term and preterm neonates admitted with clinical diagnosis of neonatal septicaemia, presenting with any one of the following features were eligible; presence of fever $\left(\geq 38.0^{\circ} \mathrm{C}\right)$ or hypothermia $\left(\leq 36.5^{\circ} \mathrm{C}\right)$, convulsions, lethargy, inability to feed, hypoglycaemia, vomiting, bulging fontanels, respiratory distress, jaundice and signs of infection on the skin (pus spots) and umbilical pus discharge or hyperaemia. Sample size in this study was calculated using Epi info version 6 statistical package, and a prevalence of $16 \%$ from a study conducted previously at the same hospital by Bloomberg et al. [10] was used with a target of recruiting about 300 participants. During the study period 1811 were admitted in the neonatal ward out of which 330 fulfilled inclusion criteria and were recruited.

Standardized questionnaires were used to obtain demographic and clinical information which included details of thorough physical examination. Weight was measured using SECA beam balance (CMS Weighing equipment, London, United Kingdom) to the nearest 100g. Two millilitres of venous blood was aseptically drawn from anterior cubital fossa of each neonate and inoculated into paediatric blood culture bottles. Pus swab were taken from the umbilical cord stump or skin pustules to determine the contribution of cord stump/skin infection to sepsis and correlate the two infections, pus swabs were transported in Stuart transport media..

Blood culture bottles were incubated at $37^{\circ} \mathrm{C}$ for $24 \mathrm{~h}$ after which aliquots were sub-cultured on solid agar plates; MacConkey, blood and chocolate agars for up $96 \mathrm{~h}$ before being regarded as no growth. Pus swabs were smeared on a glass slides for gram stain identification and were also inoculated on solid MacConkey agar at $37^{\circ} \mathrm{C}$ for $24 \mathrm{~h}$. Colonies on solid agar plates were identified based on characteristic morphology, gram stain appearance and standard commercially prepared biochemical tests [12].

Antimicrobial sensitivity testing was performed for antimicrobials which included first and second line antibiotics for treating neonatal sepsis at $\mathrm{MNH}$; first line antibiotics are ampicillin, cloxacillin and gentamicin and second line is ceftriaxone. Ampicillin $10 \mu \mathrm{g}$, cloxacillin $5 \mu \mathrm{g}$ gentamicin $10 \mu \mathrm{g}$, amikacin $30 \mu \mathrm{g}$, cefuroxime $30 \mu \mathrm{g}$, and ceftriaxone $30 \mu \mathrm{g}$ sensitivity testing were performed by Kirby Bauer diffusion method using Mueller Hinton agar with incubation of $24 \mathrm{~h}$ at $37^{\circ} \mathrm{C}$. Sensitivity was determined according to Clinical Laboratory Standard Institute standards [13]. Results were recorded as resistant, intermediate and sensitive, however, during data analysis intermediate were categorized as resistant.

Statistical Package for Social Sciences (SPSS) version 17 was used for data entering, cleaning and analysis. Chi square and Fischer's exact test were used to determine association between categorical variables. Probability (p) 
value of $<0.05$ was considered statistically significant. This study was approved by MUHAS ethical committee and informed written consents were obtained from parents/guardian prior to recruitment. Results of laboratory tests were used in the participants' management.

\section{Results}

Demographic and clinical characteristics of participants

Three hundred and thirty neonates with a median age of 3 days (range 0-26) were recruited. Hundred and sixty (48.5\%) were female and 253 (76.7\%) were aged between zero and six days. Table 1 shows demographic characteristics of participants. Most frequently reported clinical features of included pus discharge with hyperaemia (96.1\%) and fever $(91.5 \%)$. Other included inability to breastfeed, lower chest wall in-drawing, difficulty in breathing, jaundice, bulging fontanelle, skin pustules, convulsion and hypothermia (Table 1).

\section{Bacterial isolation}

Three hundred and two participants 91.5\% (302/330) had culture proven bacterial infection out of which $69.1 \%(228 / 330)$ had bacteria isolated from swabs, $1.5 \%$

Table 1 Demographic and clinical characteristics of participants

\begin{tabular}{|c|c|c|}
\hline Variables & $\mathrm{N}=330$ & $\%$ \\
\hline \multicolumn{3}{|l|}{ Age (Days) } \\
\hline $0-6$ & 253 & 76.7 \\
\hline $7-28$ & 77 & 23.3 \\
\hline \multicolumn{3}{|l|}{ Sex } \\
\hline Male & 170 & 51.5 \\
\hline Female & 160 & 48.5 \\
\hline \multicolumn{3}{|l|}{ Weight (gm) } \\
\hline$\leq 2500$ & 109 & 33 \\
\hline$>2500$ & 221 & 67 \\
\hline \multicolumn{3}{|l|}{ Maturity } \\
\hline Preterm & 77 & 23.3 \\
\hline Term & 253 & 76.7 \\
\hline \multicolumn{3}{|l|}{ Clinical features (signs \& symptoms) } \\
\hline Fever & 302 & 91.5 \\
\hline Hypothermia & 8 & 2.4 \\
\hline Inability to feed & 206 & 62.4 \\
\hline Bulging fontanelle & 33 & 10.0 \\
\hline Difficulty in breathing & 73 & 22.1 \\
\hline Lower chest wall in drawing & 89 & 27.0 \\
\hline Convulsions & 10 & 3.0 \\
\hline Umbilical pus discharge with hyperaemia & 317 & 96.1 \\
\hline Skin rash with pus spots & 31 & 9.4 \\
\hline Jaundice & 44 & 13.3 \\
\hline
\end{tabular}

(5/330) had bacteria isolated from blood and 20.9\% (69/ 330) had bacteria isolated from both swabs and blood. One hundred and ninety eight $(66.7 \%)$ swabs were taken from cord stump while 33.3\% (99/297) were taken from skin pustules. Among 69 participants who had bacteria isolated from both swabs and blood 65.2\% (45/69) had the same bacteria isolated from both swabs and blood and $34.8 \%(24 / 69)$ had different organisms isolated. Therefore culture proven sepsis was present in $22.4 \%$ (74/330).

Staphylococcus aureus was the most common isolated organism from blood culture and pus swab, followed by Klebsiella species and Escherichia coli. These three organisms accounted for approximately $85 \%$ of all isolates in blood culture and $94.3 \%$ of all isolates from pus swab. For bacteria isolated from blood Klebsiella spp $32.7 \%(17 / 52)$ was predominant at the age 0 to 6 days where as Staphylococcus aureus 54.5\% (12/22) featured more among neonates aged 7-28 days. (Table 2). Staphylococcus aureus was the predominant isolate from pus swabs in both 0-6 days $42.2 \%(98 / 323)$ and 7-28 days group $52.3 \%(34 / 65)$ (Table 3$)$.

\section{Antimicrobial susceptibility of isolated bacteria}

Antimicrobial susceptibility pattern was performed for all bacterial isolates. Isolates from both blood and pus swabs had highest resistance against ampicillin and cloxacillin. All Klebsiella spp isolates and 92.9\% (13/14) of Escherichia coli isolates from blood were resistant to ampicillin, 77.0\% (12/22) of Klebsiella spp and 42.9\% (6/14) of Escherichia coli isolated from blood were resistant to gentamycin. Twenty two (81.5\%) of Staphylococcus aureus isolated from blood were resistant to cloxacillin. Table 4 shows antimicrobial resistance pattern of organisms isolated form blood culture.

Of the isolated Klebsiella spp from swabs 97.4\% (75/77) were resistant to ampicillin and 88.7\% (63/71) of Escherichia coli isolates from swabs were resistant to ampicillin. Gentamycin resistance from Klebsiella spp and Escherichia coli were noted in 61\% (47/77) and 39.4\% (28/71) respectively. Of the Staphylococcus aureus isolates from swabs $80.3 \% \quad(106 / 132)$ were resistant to cloxacillin (Table 5).

\section{Clinical features}

Participants with fever $21.9 \%(66 / 302)$ and hypothermia $62.5 \%(5 / 8)$ were noted to have higher frequency of sepsis as compared to those with normal body temperature $15.0 \%(3 / 20), p=0.02$. Occurrence of sepsis was also noted to be higher among neonates who had skin rash with pustules $16 / 31(51.6 \%)$ as compared to those without skin rashes 58/299 (19.4\%), $\mathrm{p}<0.001$. Presence of skin rashes with pustule was an independent predicator of sepsis [OR 0.26, 95\% CI (0.10-0.66), $\mathrm{p}=0.004$ ]. 
Table 2 Bacterial isolation from blood

\begin{tabular}{|c|c|c|c|c|}
\hline \multirow[t]{2}{*}{ Organism } & \multicolumn{2}{|c|}{ Early and late infection (days) $(\mathrm{N}=74)$} & \multirow[t]{2}{*}{ Total N(\%) } & \multirow[t]{2}{*}{$\mathrm{p}$ value } \\
\hline & $0-6 n(\%)$ & $7-28 n(\%)$ & & \\
\hline Staphylococcus aureus & $15(55.6)$ & $12(44.4)$ & $27(36.5)$ & $0.721^{*}$ \\
\hline Klebsiella species & $17(77.3)$ & $5(22.7)$ & $22(29.7)$ & \\
\hline Escherichia coli & $10(71.4)$ & $4(28.6)$ & $14(18.9)$ & \\
\hline Staphylococcus epidermidis & $6(100)$ & $0(0)$ & $6(8.1)$ & \\
\hline Group B Streptococci & $1(100)$ & $0(0)$ & $1(1.4)$ & \\
\hline Pseudomonas spp & $1(50.0)$ & $1(50.0)$ & $2(2.7)$ & \\
\hline Other streptococcus species & $2(100)$ & $0(0)$ & $2(2.7)$ & \\
\hline Total & $52(70.3)$ & $22(29.7)$ & $74(100)$ & \\
\hline
\end{tabular}

*Fisher's exact test.

\section{Immediate outcome of sepsis}

Forty-six (13.9\%) neonates recruited in this study died. Thirty six neonates out of 253 (14.2\%) aged between 0-6 and 10/77 (13\%) aged between 7-28 days died. Neonates with sepsis were noted to have significantly higher proportion of deaths $18 / 74$ (24.3\%) as compared to those without sepsis $28 / 256(10.9 \%) \mathrm{p}=0.003$. No association was noted between type of isolated bacteria and death $\mathrm{p}=0.196$ (Fisher's exact test).

\section{Discussion}

From the findings of this study, neonatal sepsis is common and contributes significantly to mortality among neonates admitted in the neonatal ward at Muhimbili National Hospital. The prevalence of positive blood culture sepsis of $22.4 \%$ observed in this study is higher than $15.9 \%$ reported by Bloomberg et al. [10] from a study conducted earlier at this hospital. It is also higher than $6.5 \%$ which was reported by Klingenberg et al. [6] from a study conducted in another Tanzanian referral hospital. The increase in prevalence of sepsis could be accounted for by the increase in resistance to antimicrobial which may have resulted in inadequate treatment in lower health care facilities. Collectively, our study and that of Bloomberg et al. [10] indicate a high magnitude of neonatal sepsis at $\mathrm{MNH}$, which may reflect low quality of neonatal care as opposed to developed countries which have lower prevalence rates of neonatal sepsis $[7,14]$.

\section{Clinical outcome of neonatal sepsis}

In this study, the overall neonatal mortality was $13.9 \%$ $(46 / 330)$, being higher in neonates with sepsis $(24 \%)$ as compared to those without (10\%). Reports from other two referral hospitals in Tanzania by Kayange et al. [5] and Klingenberg et al. [6] found infant mortality due to neonatal sepsis to be $19 \%$ and matched closely with the findings of $18 \%$ by Mugalu et al. in a Ugandan study [15]. Collectively, these findings reflect the significant contribution of neonatal sepsis in both neonatal and infant mortality.

\section{Aetiology of neonatal sepsis}

We found Staphylococcus aureus, Klebsiella spp and Escherichia coli to be the predominant bacterial isolates, which is in keeping with findings from a number of studies conducted in sub-Saharan Africa [5,16-19]. Klebsiella spp was the main isolate in early onset sepsis while Staphylococcus aureus dominated in late onset sepsis and this is similar to report by Mokuolu et al. in a study which was conducted in Nigeria [17]. Organisms isolated with respect to time of onset in this study seem to fit with the proposed sources of bacteria in neonatal sepsis

Table 3 Bacterial isolation from pus swabs

\begin{tabular}{|c|c|c|c|c|}
\hline \multirow[t]{2}{*}{ Organism } & \multicolumn{2}{|c|}{ Early and late infection (days) $(\mathrm{N}=297)$} & \multirow[t]{2}{*}{ Total n (\%) } & \multirow[t]{2}{*}{ p value } \\
\hline & $0-6$ n (\%) & $7-28$ n (\%) & & \\
\hline Staphylococcus aureus & $98(74.2)$ & $34(25.8)$ & $132(44.4)$ & $0.616^{*}$ \\
\hline Klebsiella species & $61(79.2)$ & $16(20.8)$ & $77(25.9)$ & \\
\hline Escherichia coli & $57(80.3)$ & $14(19.7)$ & $71(23.9)$ & \\
\hline Staphylococcus epidermidis & $3(100)$ & $0(0)$ & $3(1.0)$ & \\
\hline Pseudomonas species & $6(85.7)$ & $1(14.3)$ & $7(2.4)$ & \\
\hline Proteus species & $7(100)$ & $0(0)$ & $7(2.4)$ & \\
\hline Total & $232(78.1)$ & $65(21.9)$ & $297(100)$ & \\
\hline
\end{tabular}

*Fisher's exact test. 
Table 4 Antimicrobial resistance pattern of isolated bacteria from blood $(\mathrm{N}=74)$

\begin{tabular}{|c|c|c|c|c|c|c|}
\hline \multirow[t]{2}{*}{ Organism } & \multicolumn{6}{|c|}{ Antibacterial agent } \\
\hline & Amp R n (\%) & Clox R n (\%) & Gent R n (\%) & Amik R n (\%) & Cefur R n (\%) & Ceft R n(\%) \\
\hline Staphy. aureus & $23(85.2)$ & $22(81.5)$ & 15 (55.6) & $0(0)$ & $4(14.8)$ & $4(14.8)$ \\
\hline Klebsiella species & $22(100)$ & $21(95.5)$ & $17(77.3)$ & $1(4.5)$ & $8(36.4)$ & $4(18.2)$ \\
\hline E. coli & $13(92.9)$ & $14(100)$ & $6(42.9)$ & $0(0)$ & $1(7.1)$ & $2(14.3)$ \\
\hline Group B Streptococci & $0(0)$ & $1(100)$ & $1(100)$ & $0(0)$ & $0(0)$ & $0(0)$ \\
\hline Pseudomonas spp & $1(50)$ & $0(0)$ & $0(0)$ & $0(0)$ & $1(50)$ & $1(50)$ \\
\hline Other Strept. species & $1(50)$ & $0(0)$ & $1(50)$ & $0(0)$ & $0(0)$ & $0(0)$ \\
\hline Total & $60(88.2)$ & $58(85.3)$ & $40(58.8)$ & $1(1.5)$ & $14(20.6)$ & $11(16.2)$ \\
\hline
\end{tabular}

Amp: Ampicillin, Clox: Cloxacillin, Gent: Gentamycin, Amik: Amikacin, Cefur: Cefuroxime, Ceft: Ceftriaxone, R: Resistance.

which are birth canal for early sepsis in which Klebsiella spp was the predominant isolate, and hospital sources or community for late sepsis in which Staphylococcus aureus was predominant isolate, respectively $[7,19]$. Staphylococcus aureus was the main isolate from swabs which may be attributed to acquisition of bacteria through handling of the neonates by health care providers and family members.

\section{Antimicrobial susceptibility of bacterial isolates}

Our findings show that most of the bacterial isolates showed high resistance to ampicillin, cloxacillin and gentamycin which are first line antimicrobials for treating neonatal sepsis at our centre. More than $80 \%$ of Staphylococcus aureus and more than $90 \%$ of Klebsiella spp from both blood and swabs were resistant to ampicillin and cloxacillin, while more than $50 \%$ of Staphylococcus aureus and more than $60 \%$ of Klebsiella spp were resistant to gentamycin. High resistance noted may be attributed to excessive and irrational use of these antibiotics at primary health facilities from which neonates are referred to our centre.

Notably, the resistance of all isolates to ceftriaxone, cefuroxime and amikacin was significantly low. At $\mathrm{MNH}$ ceftriaxone is used a second line, while cefuroxime and amikacin are not included in the protocol of neonatal sepsis management. From our findings and other studies in the region showing high levels of antimicrobial resistance to ampicillin, cloxacillin and gentamycin $[6,18,20]$ it is apparent that the current antimicrobial regiment for empirical treatment of neonatal sepsis need to be revisited. Amikacin and cefuroxime may be alternative choice of antimicrobials in empiric treatment of neonatal sepsis however these will be more expensive.

\section{Study limitations}

Some of the study participants referred to our hospital might have been inadequately treated resulting in selection bias in our study. Anaerobes which may cause sepsis and other infections with similar presentation to sepsis including malaria [21] were not looked for in this study. Staphylococcus epidermidis isolated in early neonatal sepsis $6 / 52(11.5 \%)$ may be due to contamination during blood collection [22,23]. Extended beta lactamase resistant strains were not determined and resistance to methicillin by Staphylococcus aureus isolates were not determined in this study.

\section{Conclusions}

Staphylococcus aureus and Klebsiella spp were the predominant bacterial isolates in this study and showed high resistance to ampicillin and cloxacillin which are WHO recommended and routinely used antimicrobial at $\mathrm{MNH}$. This calls for institutional based antimicrobial surveillance to guide choices of antibiotics for treating neonatal sepsis.

Table 5 Antimicrobial resistance pattern of isolated bacteria from swabs ( $N=2974)$

\begin{tabular}{|c|c|c|c|c|c|c|}
\hline \multirow[t]{2}{*}{ Organism } & \multicolumn{6}{|c|}{ Antibacterial agent } \\
\hline & Amp R n (\%) & Clox R n (\%) & Gent R n (\%) & Amik R n (\%) & Cefur R n (\%) & Ceft R n (\%) \\
\hline Staphy aureus & $116(87.9)$ & $106(80.3)$ & $69(52.3)$ & $7(5.3)$ & $45(34.1)$ & $55(41.7)$ \\
\hline Kleb species & 75 (97.4) & 75 (97.4) & $47(61)$ & $2(2.6)$ & $34(44.2)$ & $31(40.3)$ \\
\hline E. coli & $63(88.7)$ & $60(84.5)$ & $28(39.4)$ & $5(7)$ & $24(33.8)$ & $19(26.8)$ \\
\hline Pseudomonas spp & $7(100)$ & $7(100)$ & $4(57.1)$ & $0(0)$ & $4(57.1)$ & $6(85.7)$ \\
\hline Proteus species & $6(85.7)$ & $5(71.4)$ & $2(28.6)$ & $0(0)$ & $0(0)$ & $2(28.6)$ \\
\hline Total & $267(90.8)$ & $253(86.1)$ & $150(51.0)$ & $14(4.8)$ & $107(36.4)$ & $113(38.4)$ \\
\hline
\end{tabular}

Amp: Ampicillin, Clox: Cloxacillin, Gent: Gentamycin, Amik: Amikacin, Cefur: Cefuroxime, Ceft: Ceftriaxone, R: Resistance. 


\section{Competing interests}

Authors declare that they have no competing interests.

\section{Authors' contributions}

TVM designed the study, collected data, analyzed data and participated in manuscript preparation. FF assisted in data collection, analysis and prepared the manuscript. MIM and AM critically reviewed the study protocol, supervised data collection and participated in manuscript preparation. All authors have read and approved the final manuscript.

\section{Acknowledgements}

Authors are grateful to the nurses in the neonatal ward of Muhimbili National Hospital for facilitating data collection. Special thanks to laboratory technicians in the Department of Microbiology and Immunology, MUHAS for carrying out laboratory tests. Ministry of Health and Social Welfare of Tanzania is acknowledged for funding this research work.

\section{Author details}

'Department of Paediatrics, Bugando Medical Centre, P O Box 1370, Mwanza, Tanzania. ${ }^{2}$ Department of Paediatrics and Child Health, School of Medicine, Muhimbili University of Health and Allied Sciences, P O Box 65001, Dar es Salaam, Tanzania. ${ }^{3}$ Department of Microbiology and Immunology, School of Medicine, Muhimbili University of Health and Allied Sciences, P O Box 65001, Dar es Salaam, Tanzania.

Received: 30 May 2012 Accepted: 22 October 2012

Published: 24 October 2012

\section{References}

1. Lawn JE, Cousens S, Zupan J: 4 million neonatal deaths: when? where? why? Lancet 2005, 365:891-900.

2. Vergnano S, Sharland M, Kazembe P, Mwansambo C, Heath PT: Neonatal sepsis: an international perspective. Arch Dis Child Fetal Neonatal Ed 2005, 90:220-24.

3. Seale AC, Mwaniki M, Newton CRJC, Berkley JA: Maternal and early onset neonatal bacterial sepsis: burden and strategies for prevention in sub-Saharan Africa. Lancet Infect Dis 2009, 9(7):428-438.

4. Shalini Tripathi S, Malik GK: Neonatal Sepsis: past, present and future; a review article. Inter J Med Up 2010, 5(2):45-54

5. Kayange N, Kamugisha E, Mwizamholya DL, Jeremiah S, Mshana SE: Predictors of positive blood culture and death among neonates with suspected neonatal sepsis in a tertiary hospital, Mwanza-Tanzania. BMC Pediatr 2010, 10:39.

6. Klingenberg C, Olomi R, Oneko M, Sam N, Langeland N: Neonatal morbidity and mortality in a Tanzanian tertiary care hospital. Ann Trop Paediatr 2003, 23(4):293-9.

7. Edmund K, Zaidi A: New approaches to preventing, diagnosing and treating neonatal sepsis. PLoS Med 2010, 7(3):e1000213.

8. Qazi AS, Stoll BJ: Neonatal sepsis- A major global public health challenge. Ped Infect Dis J 2009, 28:1.

9. Bradford PA: Extended spectrum beta lactamases in the $21^{\text {st }}$ century-Characterization epidemiology and detection of this important resistance threat. Clin Microbiol Rev 2001, 14:933-951.

10. Blomberg B, Manji KP, Urassa WK, et al: Antimicrobial resistance predicts death in Tanzanian children with bloodstream infections: a prospective cohort study. BMC Infect Dis 2007, 7:43.

11. WHO: Integrated Management of Childhood Illnesses Handbook. Geneva: 2005. http://whqlibdoc.who.int/publications/2005/9241546700.pdf. ISBN 9241546700 (NLM classification: WS 29).

12. Barrow Gl, Feltham RKA: Cowan and Steel's Manual for Identification of Medical Bacteria. 3rd edition. Cambridge: Cambridge University Press; 2003.

13. Clinical and Laboratory Standard Institute: Performance standards for antimicrobial disk susceptibility tests. Approved standard. In Ninth edition document M2-A9. Wayne: Clinical and Laboratory Standards Institute; 2006.

14. World Health Organization: Essential Newborn Care. In A report of Technical Working Group. Geneva: WHO; 1995. https://apps.who.int/rht/documents/ MSM96-13/essential_newborn_care.htm.

15. Mugalu J, Nakakeeto MK, Kiguli S, Kaddu-Mullindwa DH: Aetiology, risk factors and immediate outcome of bacteriologically confirmed neonatal septicaemia in Mulago hospital, Uganda. Afr Health Sci 2006, 6:120-126.
16. Iregbu KC, Elegba OY, Babaniyi IB: Bacteriological profile of neonatal septicemia in a tertiary hospital in Nigeria. Afr Health Sci 2006, 6(3):151-154.

17. Mokuolu AO, Jiya N, Adesiyun OO: Neonatal septicemia in llorin: bacteria pathogens and antibiotic sensitivity pattern. Afr J Med Med Sci 2002, 31:127-130.

18. Shitaye D, Asrat D, Woldeamanuel Y, Worku B: Risk factors and etiology of neonatal sepsis in Tikur Anbessa University Hospital, Ethiopia. Ethiop Med J 2010, 48(1):11-21.

19. Newton O, English M: Young infant sepsis: aetiology, antibiotic susceptibility and clinical signs. Trans R Soc Trop Med Hyg 2007, 101(10-4):959-966.

20. Musoke RN, Revathi G: Emergence of multidrug-resistance gram-negative organisms in a neonatal unit and therapeutic implications. $J$ Trop Pediatr 2000, 46(2):89-91.

21. Mwaniki MK, Talbert AW, Mturi FN, Berkeley JA, Kager P, Marsh K, Newton CR: Congeital and neonatal malaria in a rural Kenyan district hospital: An eight year analysis. Malaria J 2010, 9:313.

22. Hasemizadeh Z, Bazargani A, Davarpanah AM: Blood culture contamination in a neonatal intensive care unit in Shiraz, Southwest-Central Iran. Med Princ Pract 2011, 20:133-6.

23. Wolkowiez MC, Moran C, Benjamin DK, Cotton CM, Clark RH, Benjamin DK $\mathrm{Jr}$, Smith B: Early and late sepsis in late preterm infants. Paed Infect Dis J 2009, 28(12):1252-6.

doi:10.1186/1471-2458-12-904

Cite this article as: Mhada et al: Neonatal sepsis at Muhimbili National Hospital, Dar es Salaam, Tanzania; aetiology, antimicrobial sensitivity pattern and clinical outcome. BMC Public Health 2012 12:904.

\section{Submit your next manuscript to BioMed Central and take full advantage of:}

- Convenient online submission

- Thorough peer review

- No space constraints or color figure charges

- Immediate publication on acceptance

- Inclusion in PubMed, CAS, Scopus and Google Scholar

- Research which is freely available for redistribution 